\title{
Investigation of the electronic properties of cyclopentadithiophene polymers and copolymers
}

Paolo Coppo* and Michael L. Turner*

Department of Chemistry, University of Sheffield, Dainton Building, Brook Hill, S3 7HF, Sheffield, UK.

Domenico C. Cupertino and Stephen G. Yeates

Avecia Research Centre, Hexagon Tower, Blackley, M9 8ZS, Manchester, UK.

\begin{abstract}
Cyclopentadithiophene based homopolymers and copolymers have been investigated as semiconductor layers in field effect transistors. They generally show low hole mobilities due to a disordered amorphous structure in the solid state. FET mobility can be enhanced in the homopolymer, although this results in poor on/off ratio of the device. The low mobilities may be attributed to the presence of solubilizing alkyl chains normal to the long axis of the polymer which prevent the self assembly of the polymer into an ordered $\pi$-stacked configuration.
\end{abstract}

\section{INTRODUCTION}

Solution processable conjugated polymers have attracted considerable attention due to their possible application in low performance plastic circuits.[1] Tremendous recent interest has focused on understanding the mechanisms of charge transport in conjugated polymers, and it is clear that the electronic properties of thin films of these materials are strongly dependent on the degree of order achieved in the solid state. Conjugated polymers that form disordered amorphous thin films show low charge mobilities due to inefficient inter-chain transport of carriers.[2] To achieve higher charge mobilities in thin films of polymers, enhanced inter-chain order is required. This is seen for thin films of poly(3-alkylthiophenes) as these polymers can self assemble in the solid state when processed from solution.[3] In particular, the polymer chains are aligned such that the $\pi$-stacking direction is parallel to the substrate, allowing fast transport of the carriers in the transistor channel. The mobility of holes can be as high as $0.1 \mathrm{~cm}^{2} / \mathrm{Vs}$ in a field effect transistor.[4] Self-assembly from liquid crystal phases has also been observed for a dioctylfluorene-bithiophene copolymer (F8T2). This polymer can be aligned from the nematic phase and quenched into a quasi-monodomain that showed enhanced mobility, up to 0.01 $\mathrm{cm}^{2} /$ Vs. [5]

In this contribution, polycyclopentadithiophenes, a class of solution processable, thiophene based analogues of the polyfluorenes [6], are investigated as charge transport materials in FETs.

\section{RESULTS AND DISCUSSION:}

Dioctylcyclopentadithiophene homopolymer (1) was synthesised via oxidative polymerization using iron(III) chloride, according to previously reported procedures.[7] Two different samples, containing a different amount of iron impurities were tested. In the first sample (1a), $1050 \mathrm{ppm}$ of iron were detected by ICP-AES analysis. A thin film of this material, processed by spin casting from a $0.8 \%$ chloroform solution, gave a FET mobility of $2 \times 10^{-3}$ $\mathrm{cm}^{2} /$ Vs. Further purification by filtration through a silica gel layer gave a second sample (1b) 
containing a lower level of iron impurities (145 ppm). Thin films of this material showed a lower mobility, $7.9 \times 10^{-5} \mathrm{~cm}^{2} / \mathrm{Vs}$, indicating that the level of iron impurities strongly affects the mobility. Furthermore, the on/off ratio for devices fabricated from 1a was lower than 10, due to a high off current. The low mobility of thin films of polymer $\mathbf{1 b}$ indicated that the solution processed polymer films are disordered and amorphous. This observation is consistent with the absence of any identifable thermal transitions in the DSC analyses. [8] Indeed, the optical properties are also typical of amorphous polymers, as they show no red shift in the UV-visible absorption spectra when passing from solution to the solid state.[7b],[9] The difference between the solid state morphology of poly(3-alkylthiophenes)and the polycyclopentadithiophenes is presumably due to the presence of solubilizing alkyl chains normal to the polymer backbone that inhibit self ordering into a $\pi$-stacked configuration.

Alternating copolymers (3) of cyclopentadithiophene and dioctylfluorene repeat units were prepared by Suzuki cross coupling and thin films of these polymers were tested against a reference sample of F8T2 prepared with the same procedure.[10] These polymers show low molecular weight, when compared to that of polymer $\mathbf{1}$. The reference material F8T2 (2) shows a field effect mobility of $1.5 \times 10^{-3}$ when spin coated from toluene, without any alignment technique. The cyclopentadithiophene copolymers ( 3 and $\mathbf{4})$ were spin coated from toluene solutions but their mobility was found to be several orders of magnitude lower. Polymer $\mathbf{3}$ shows a field effect mobility of $10^{-7} \mathrm{~cm}^{2} / \mathrm{Vs}$ and polymer 4 shows a field effect mobility of $5 \times 10^{-6}$ $\mathrm{cm}^{2} / \mathrm{Vs}$.

\begin{tabular}{|c|c|c|c|}
\hline & $\begin{array}{c}\text { UV-vis. } \\
\lambda_{\max }(/ \mathrm{nm})\end{array}$ & $\begin{array}{c}\text { FET mobility } \\
\left(/ \mathrm{cm}^{2} / \mathrm{Vs}\right)\end{array}$ \\
\hline $\mathrm{M}_{\mathrm{w}} / \mathrm{M}_{\mathrm{n}}$ & 600 & $2 \times 10^{-3}$ \\
\end{tabular}

Table 1. Chemical structure, solution molecular weight determined by GPC against polystyrene standards, UV-vis absorption maxima and FET mobility for polymers 1-4.

In polymer 3 the presence of solubilizing chains on both repeat units may inhibit the stacking of adjacent chains. However, for polymer 4 the structure is very similar to that of F8T2, 
where no steric impediment is present on the thiophene units. Direct observation of the films by means of polarized light microscopy reveals that a high degree of crystallinity characterizes F8T2 (2), which melts into a nematic phase upon heating at $200^{\circ} \mathrm{C}$ (see figure 1). Conversely, films of polymer $\mathbf{4}$ are amorphous. Heating a polymer film of polymer $\mathbf{4}$ over $200^{\circ} \mathrm{C}$ resulted in degradation with no liquid crystal phase observable and this was confirmed the absence of any identifable thermal transitions by DSC.

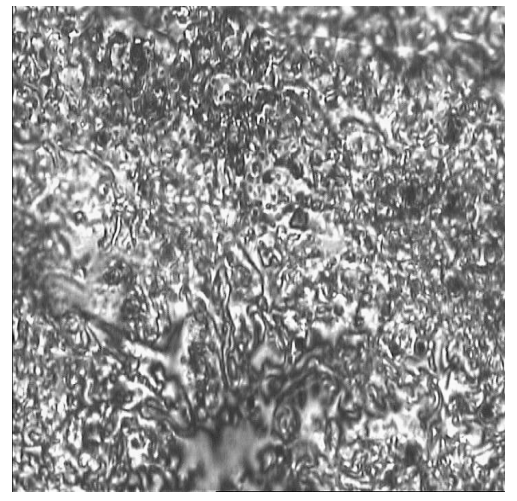

Figure 1. nematic phase in $\mathrm{F} 8 \mathrm{~T} 2\left(200^{\circ} \mathrm{C}\right)$

\section{CONCLUSIONS}

In conclusion, cyclopentadithiophene homopolymers and copolymers were tested as charge transport layers in FET devices. They show a low mobility, due to a disordered amorphous structure in the solid state. FET mobility can be enhanced in the homopolymer, although this results in poor on/off ratio of the device.

\section{ACKNOWLEDGEMENTS}

We wish to acknowledge Avecia Ltd., The Royal Society and the EPSRC for funding this project. Dr J. Veres and Dr S. Ogier are gratefully acknowledged for the FET measurements.

\section{EXPERIMENTAL DETAILS}

All experiments were carried out under nitrogen. Polymers were prepared, dedoped and purified according to the procedured previously reported. [7b] Chloroform and toluene used for the polymers casting were purchased from Fisher chemicals (HPLC grade). Anhydrous tetrahydrofuran and diethyl ether were obtained by distillation over sodium/benzophenone ketyl. All other reagents were used as received. ${ }^{1} \mathrm{H}$ and ${ }^{13} \mathrm{C}$ NMR spectra were recorded using a Bruker AC $250 \mathrm{MHz}$ spectrometer operating at $250.133 \mathrm{MHz}$ for ${ }^{1} \mathrm{H}$ NMR spectroscopy and a Bruker AMX $400 \mathrm{MHz}$ spectrometer operating at $100.624 \mathrm{MHz}$ for ${ }^{13} \mathrm{C}$ NMR spectroscopy. Molecular weight determination was carried out in chloroform solution on an HP1090 liquid chromatograph using Polymer Laboratories Mixed C or Mixed E chromatography columns that were calibrated against narrow molecular weight polystyrene calibration standards. $\mathrm{CHN}$ analysis was determined using a Perkin Elmer 2400 CHN Elemental Analyser. Mass spectra were recorded on a Micromass Promass spectrometer. UV / Vis spectra were recorded on a Unicam UV / Vis 4 
spectrophotometer. Polarized light microscopy was carried out with an Olympus BH-2 optical microscope equipped with a Linkam THM600 hotstage and a PR600 control unit. Differential scanning calorimetry (DSC) traces were recorded on a Perkin Elmer Pyris 1.

FET devices were fabricated according to the specifications reported elsewhere.[11]

\section{REFERENCES}

1. T. A. Skotheim, R. L. E. Elsenbaumer, J. R. Reynolds editors, Handbook of conducting polymers, second edition revised and expanded, 1997, p. 1112.

2. J. Cornil, D. Beljonne, J. P. Calbert, J. L. Brédas, Adv. Mater., 13, 1053 (2001).

3. H. Sirringhaus, P. J. Brown, R. H. Friend, M. M. Nielsen, K. Bechgaard, B. M. W. Langeveld-Voss, A. J. H. Spiering, R. A. J. Janssen, E. W. Meijer, P. Herwig, D. M. de Leeuw, Nature, 401, 685 (1999).

4. H. Sirringhaus, N. Tessler, R, H. Friend, Science, 280, 1741 (1998).

5. H. Sirringhaus, R. J. Wilson, R. H. Friend, M. Inbasekaran, W. Wu, E. P. Woo, M. Grell, D. D. C. Bradley, Appl. Phys. Lett., 77, 406 (2000).

6. G. Zotti, G. Schiavon, A. Berlin, G. Fontana, G. Pagani, Macromolecules, 27, 1938 (1994).

7. a) P. Coppo, D. C. Cupertino, S. G. Yeates, M. L. Turner, J. Mater. Chem., 12, 2597 (2002). b) P. Coppo, D. C. Cupertino, S. G. Yeates, M. L. Turner, Macromolecules, 36, 2705 (2003).

8. P. Coppo, M. L. Turner, unpublished results.

9. R. D. McCullough, Adv. Mater., 10, 93 (1998).

10. M. Inbasekaran, W. Wu, E. P. Woo, US patent, 5,777,070, 1998, 9pp.

11. J. Veres, S. D. Ogier, S. W. Leeming, D. C. Cupertino S. M. Khaffaf, Adv. Funct. Mater., 13, 199 (2003). 